\title{
Dynamics of SMA micro-actuator in biomechanical system
}

\author{
Rafal Rusinek ${ }^{1, \star}$, Andrzej Weremczuk ${ }^{1, \star \star}$, Marcin Szymanski ${ }^{2, \star \star \star}$, and Jerzy Warminski ${ }^{1, \star \star \star \star}$ \\ ${ }^{1}$ Lublin University of Technology, Nadbystrzycka 36, 20-618 Lublin, Poland \\ ${ }^{2}$ Medical University of Lublin, Jaczewskiego 8, 20-854 Lublin, Poland
}

\begin{abstract}
In this paper the polynomial model of shape memory alloy is used to characterise properties of a micro-actuator which is applied as a new middle ear prosthesis. A two degrees of freedom model of the reconstructed middle ear is solve by means of multiple time scales method. The system has various behaviours near the primary resonance depending on ambient temperature. The special case when relative temperature $\theta=1.0$ characterises untypical resonance curve. Increasing temperature to the normal human body one the resonance curves are typical. Then the system has only one periodic solution if the excitation is not too strong.
\end{abstract}

\section{Introduction}

Shape memory alloys (SMAs) are a family of metals with the ability to change shape depending on their temperature [1]. SMA may exhibit one or two-way shape memory effect which is attractive in various science and engineering applications starting from biomedical and ending to aerospace industry. SMA is used to built thermal actuators, stabilizing mechanisms and so on. SMAs undergo thermo-elastic phase transformations between martensite and austenite induced by temperature or stress. Two variants of the martensitic phase are possible: twinned or detwinned. The conversion from twinned to detwinned martensite takes place by means of loading process. When the loading - unloading process is finished, some amount of residual strain remains, meaning that the reverse transformation from detwinned to twinned martensite is not completed. The shape memory effect appears by heating which activates the transformation from detwinned martensite to austenite. When SMA is stressed at temperatures that are above the austenite transformation temperature an inelastic deformation (hysteresis loop) is observed. This phenomenon is known as pseudoelastic effect [2, 3].

These thermomechanical properties of SMAs are studied in literature by microscopic or macroscopic models. The microscopic models treat phenomena on a molecular level, therefore the macroscopic phenomenological models are more attractive from practical point of view. In the literature one can find a lot of interesting and thorough description of SMAs [4-10] and their applications [11-14]. Different models of SMA give various results depending on nonlinear phenomenon which are taken into account. A comparison of popular SMA models is presented in [15].

\footnotetext{
$\star$ e-mail: r.rusinek@pollub.pl

$\star \star$ e-mail: a.weremczuk@pollub.pl

$\star \star \star$ e-mail: marcinszym@onet.pl

$\star \star \star \star$ e-mail: j.warminski@pollub.pl
}

In this paper the model with polynomial free-energy based on Devonshire theory is used to model SMA micro actuator. Temperature induced phase transition, combined with hysteresis was proposed by Falk [16] and Falk and Konopka [17]. In that model free energy depends on the temperature and the one-dimensional strain. On the other hand, the SMA micro actuator in the form of prosthesis is applied to reconstruct a damaged human middle ear. The prosthesis made of SMA is able to change its shape and length while being heated and remain unchanged after cooling. Therefore, the SMA micro actuator could provide better sound transfer conditions from an outer to an inner ear. The idea of using SMA to build middle ear prosthesis is presented in [18-20]. However nowadays, a typical middle ear prosthesis is made of titanium. Its size and shape is widely discussed in literature e.g. [21-24].

Here, in this contribution an analysis of the reconstructed middle ear (RME) by means of the SMA micro actuator (shape memory prosthesis - SMP) is presented. A two degrees of freedom (2dof) model of the RME is solved analytically with the help of the multiple times scale method (MTSM). Finding the resonance curves at different temperatures is the main goal of the paper.

\section{Modelling}

The human middle ear composed of three parts: the outer, the middle and the inner ear. Sound is transmitted from the outer to the inner through the middle ear which is goal of the present analysis. The middle ear consists of three small bones: the malleus, the incus and the stapes connected each other. When the ossicular chain structure is destroyed by inflammatory diseases such as chronic suppurative otitis media or cholesteatoma, the middle ear should be reconstructed. In the medical practice it is called ossiculoplasty (the reconstruction of the middle ear ossicles). For over 50 years, middle ear surgery techniques 
have improved hearing that had been destroyed by various diseases. Numerous procedures are currently used in clinical practice, and a variety of middle ear prostheses made of titanium or titanium alloy are available. Most prostheses can be modified only once they have been prepared. If a prosthesis is too short, it cannot be remodelled. However, prostheses of different sizes can be chosen or tailored by a surgeon to the desired length. Their length can be adjusted by cutting the prosthesis leg. To improve and fasten implementation process we propose a shape memory prosthesis (SMP) that can adjust its size and angulation to the requirements of a particular medical case. The prosthesis can be made of the Nitinol with one-way shape memory effect. It must change its shape while being heated and remain unchanged after cooling. In medical practice, when the middle ear is damaged usually the incus has to be removed to implement a prosthesis between the malleus and the stapes. Therefore 2 dof model of reconstructed middle ear is proposed and characterised in Sect. 2.2. The mathematical model of the SMA element is presented in Sect. 2.1.

In the present work, we focus on analytical solutions of the nonlinear system with SME which allows wide range parametric studies for any mechanical and biomechanical systems. However, the detailed investigations are performed for the parameters corresponding to the model of a reconstructed human middle ear.

\subsection{SMA actuator}

The SMA actuator (prosthesis) is modelled by a polynomial dependence developed by Falk [16]. The spring force of the prosthesis can be written as

$$
F_{S M A}=\sigma A
$$

where $A$ is a cross-section of the prosthesis. The stress $\sigma$ is described by a polynomial proposed by Falk [16] and used to describe the pseudoelasticity and shape memory effect as well [25]. The stress of the SMA is given by

$$
\sigma=a\left(T-T_{M}\right) \varepsilon-b \varepsilon^{3}+\frac{b^{2}}{4 a\left(T_{A}-T_{M}\right)} \varepsilon^{5}
$$

where, $\varepsilon$ is a strain, $T$ means temperature, $a$ and $b$ are positive material constants. $T_{M}$ and $T_{A}$ mean the temperature when martensite (subscript $M$ ) and austenite (subscript $A$ ) is stable. Finally, after calculations the force of SMA prosthesis is defined as

$$
\begin{array}{r}
F_{S M A}= \\
a_{1}\left(T-T_{M}\right)\left(x_{P}-x_{S}\right)-a_{2}\left(x_{P}-x_{S}\right)^{3}+a_{3}\left(x_{P}-x_{S}\right)^{5}
\end{array}
$$

where, $x_{P}$ and $x_{S}$ mean displacements of the masses in the model presented in Fig.1.

\subsection{Middle ear with SMA actuator}

The 2dof model of RME consists of two lumped masses, springs and dampers as shown in (Fig.1). In the model the stapes $\left(m_{S}\right)$ and the malleus $\left(m_{M}\right)$ are connected each other by the SME (in this case called also as the shape memory prosthesis). The tympanic membrane, the cochlea and the annular ligament stiffness and damping are defined as $k_{T M}$, $k_{C}, k_{A L}, c_{T M}, c_{C}$ and $c_{A L}$, respectively. The micro-actuator

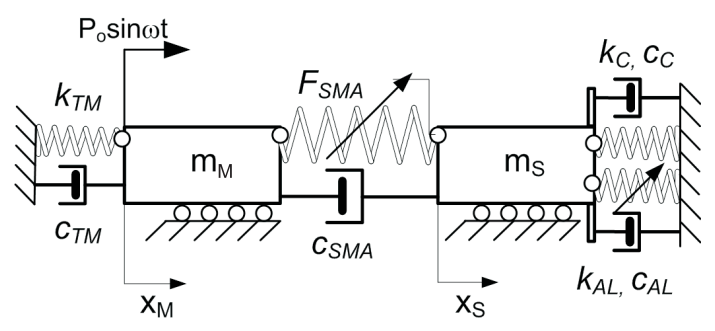

Figure 1. Model of a middle ear with SMA prothesis

as a SMA spring $\left(F_{S M A}\right)$ is described by Eq. 3. The differential equations of motion of the system with the SMA micro-actuator take the form

$$
\begin{array}{r}
m_{M} \ddot{x}_{P}+k_{T M} x_{M}+F_{S M A}+c_{T M} \dot{x}_{M}=P_{0} \sin \omega \tau \\
m_{S} \ddot{x}_{S}+k_{C} x_{S}+k_{A L} x_{S}+k_{2 A L} x_{S}{ }^{2}+k_{3 A L} x_{S}{ }^{3}+ \\
c_{C} \dot{x}_{S}+c_{A L} \dot{x}_{S}-c_{S M A}\left(\dot{x}_{M}-\dot{x}_{S}\right)-F_{S M A}=0
\end{array}
$$

The model is solved analytically by means of the multiple time scales method in Sect. 3

\section{Analytical solution}

The dimensional equations of motion (Eq.4) is transformed to the nondimensional, and next the quasi-normal coordinates and linear eigenvalue problem is solved. Then, an approximate analytical solution is determined by means of the multiple time scale method [26]. According to this method the solution is expressed by series of a small parameter $\varepsilon$

$$
\eta_{j}(\tau, \varepsilon)=\eta_{j 0}\left(T_{0}, T_{1}\right)+\varepsilon \eta_{j 1}\left(T_{0}, T_{1}\right)+\ldots j=1,2
$$

Finally, the equation of the resonance curve near the first natural frequency has the form

$$
\begin{array}{r}
\frac{9}{16} \alpha_{2}^{2} a_{1}^{6}+\frac{3}{2}\left(\omega_{01}^{2}-\omega^{2}\right) \alpha_{2} a_{1}^{4}+ \\
{\left[\zeta_{1}^{2} \omega^{2}-\left(\omega_{01}^{2}-\omega^{2}\right)^{2}\right] a_{1}^{2}-p_{1}^{2}=0}
\end{array}
$$

The more detailed description of the methodology and transformation from dimensional parameters shown in Eqs. (3)-(4) and the dimensionless presented in Eq.(6) can be found in [27]. Eq. (6) is used to draw the primary resonance curves (Fig.2) for two variants of relative temperature $\theta=1$ and $\theta=1.0787$ which relates to the normal temperature of the human body. Moreover, the excitation amplitude varies from $P_{0}$ to $10 P_{0}$. Black colour depicts stable while red - unstable periodic solutions. For the calculations the following set of parameters is used [27]:

$$
\begin{gathered}
m_{P}=2.5525 \times 10^{-5} \mathrm{~kg}, m_{S}=1.78 \times 10^{-6} \mathrm{~kg}, \\
k_{T M}=300 \mathrm{~N} / \mathrm{m}, k_{C}=200 \mathrm{~N} / \mathrm{m}, k_{A L}=623 \mathrm{~N} / \mathrm{m},
\end{gathered}
$$



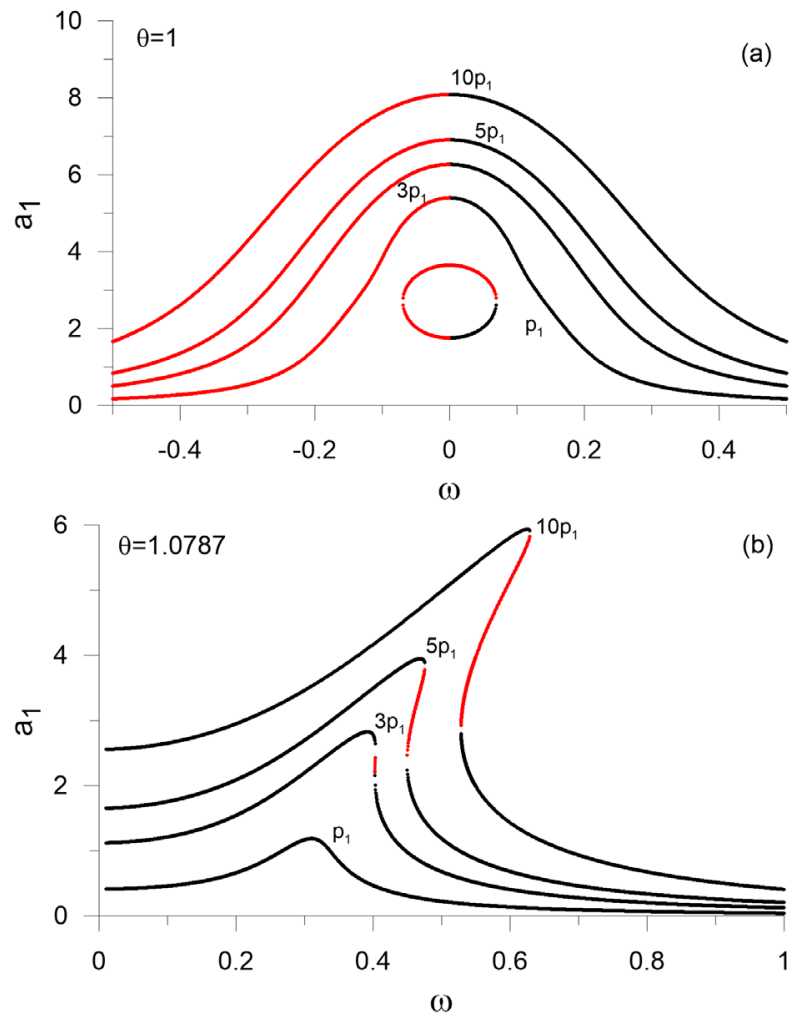

Figure 2. Primary resonance curves, $\theta=1$ (a) and $\theta=1.0787$ (b)

$$
\begin{gathered}
k_{A L 2}=3 \times 10^{8} \mathrm{~N} / \mathrm{m}^{2}, k_{A L 3}=2.25 \times 10^{15} \mathrm{~N} / \mathrm{m}^{3}, \\
a_{1}=157080 \mathrm{~kg} /(\mathrm{Km}), a_{2}=2.5233 \times 10^{14} \mathrm{~kg} /(\mathrm{ms})^{2}, \\
a_{3}=3.8666 \times 10^{21} \mathrm{~kg} /\left(\mathrm{m}^{2} \mathrm{~s}\right)^{2}, T_{M}=287.0 \mathrm{~K}, \\
P_{0}=1.2 * 10^{-4} \mathrm{~N}, c_{T M}=0.06 \mathrm{~kg} / \mathrm{s}, \\
c_{S M A}=0.055 \mathrm{~kg} / \mathrm{s}, c_{C}=0.0017 \mathrm{~kg} / \mathrm{s}, c_{A L}=0.00004 \mathrm{~kg} / \mathrm{s} .
\end{gathered}
$$

When the relative temperature is low $\theta=1.0$ the system resonance curve is untypical. This is because at $\theta=1.0$, $T-T_{M}=0$. For excitation $P_{0}$ a circle appears in the middle. Then two stable solutions are possible. For larger excitation amplitude only one stable solution exists. In case of $\theta=1.0787$ the system behaves typically. For excitation $P_{0}$ the system is fully predictable because it has only one solution, that is important from practical point of view. Larger excitation can lead to two stable solution. This situation is not wanted in the middle ear.

\section{Conclusions}

The micro-actuator proposed here as the prosthesis of the human middle ear has various behaviour depending on temperature. The special case when $\theta=1.0$ characterises untypical resonance curve. Increasing temperature to the normal human body one the resonance curves are typical. Then the system has only one periodic solution if the excitation is not too strong. That is a positive aspect from practical point of view. More examples and information about primary resonance can be found in [27] where the problem of the reconstructed middle ear by means of the shape memory prosthesis is discussed.

\section{References}

[1] M.A. Savi, P.M. Pacheco, A.M. Braga, International Journal of Non-Linear Mechanics 37, 1387 (2002)

[2] M.A. Savi, A.M.B. Braga, Journal of the Brazilian Society of Mechanical Sciences and Engineering 15, 1 (1993)

[3] M.A. Savi, M.A. Sá, A. Paiva, P.M. Pacheco, Chaos, Solitons \& Fractals 36, 828 (2008)

[4] L.C. Brinson, Journal of Intelligent Material Systems and Structures 4, 229 (1993)

[5] F. Auricchio, A. Reali, Materials Science Forum 583, 257 (2008)

[6] V. Piccirillo, J.M. Balthazar, A.M. Tusset, D. Bernardini, G. Rega, Proceedings of the Institution of Mechanical Engineers, Part C: Journal of Mechanical Engineering Science 230, 120 (2015)

[7] de Aguiar, Ricardo Alexandre Amar, de Castro Leão Neto, Waldyr Cardoso, M.A. Savi, Calas Lopes Pacheco, Pedro Manuel, Materials Science Forum 758, 147 (2013)

[8] A. Rao, A. Ruimi, A.R. Srinivasa, International Journal of Solids and Structures 51, 4554 (2014)

[9] J.H. Roh, Mathematical Problems in Engineering 2014, 1 (2014)

[10] M. Fabrizio, M. Pecoraro, V. Tibullo, Mechanics Research Communications 74, 20 (2016)

[11] E. Nikbakht, K. Rashid, F. Hejazi, S.A. Osman, Structure and Infrastructure Engineering 11, 297 (2014)

[12] Z.W. Zhu, Y.N. Liu, J. Xu, H.L. Wang, Modeling and application of Shape Memory Alloy based on hysteretic nonlinear theory and data mining, in $20083 \mathrm{rd}$ International Conference on Intelligent System and Knowledge Engineering (IEEE, 2008), pp. 329-334, ISBN 978-1-4244-2196-1

[13] L.G. Machado, M.A. Savi, Pacheco, Pedro Manuel C.L., International Journal of Solids and Structures 40, 5139 (2003)

[14] D. Bernardini, G. Rega, Mathematical and Computer Modelling of Dynamical Systems 11, 291 (2005)

[15] D. Bernardini, G. Rega, International Journal of Mechanical Sciences 130, 458 (2017)

[16] F. Falk, Acta Metallurgica 28, 1773 (1980)

[17] F. Falk, P. Konopka, Journal of Physics: Condensed Matter 2, 61 (1990)

[18] R. Rusinek, J. Warminski, M. Szymanski, K. Kecik, K. Kozik, International Journal of Mechanical Sciences 127, 163 (2017)

[19] R. Rusinek, M. Szymanski, J. Warminski, Journal of Computational and Nonlinear Dynamics (2017)

[20] J. Latalski, R. Rusinek, The European Physical Journal Plus 132, 645 (2017)

[21] K.D. Shah, R.A. Bradoo, A.A. Joshi, D.D. Sapkale, Indian Journal of Otolaryngology and Head \& Neck Surgery 65, 298 (2013)

[22] S. Şevik Eliçora, D. Erdem, A.E. Dinç, M. Damar, S. Bişkin, European Archives of Oto-Rhino- 
Laryngology 274, 773 (2017)

[23] A. Coordes, L. Jahreiss, U. Schönfeld, M. Lenarz, The Laryngoscope 127, 500 (2017)

[24] O. Fawole, S.E. Mowry, American Journal of Otolaryngology 37, 136 (2016)

[25] A. Paiva, M.A. Savi, Mathematical Problems in Engineering 2006, 1 (2006)
[26] A.H. Nayfeh, Perturbation methods (Wiley, New York, 1973), ISBN 9780471630593

[27] R. Rusinek, J. Warminski, A. Weremczuk, M. Szymanski, International Journal of Non-Linear Mechanics (under review) (2018) 